\title{
BURST-BY-BURST ADAPTIVE WIRELESS VIDEO TELEPHONY OVER DISPERSIVE CHANNELS
}

\author{
C.H. Wong, P.J. Cherriman, L. Hanzo \\ Dept. of Electronics and Computer Science, Univ. of Southampton, SO17 1BJ, UK. \\ Tel: +44-703-593 125, Fax: +44-703-593 045 \\ Email: pjc@ecs.soton.ac.uk, lh@ecs.soton.ac.uk \\ http://www-mobile.ecs.soton.ac.uk
}

\begin{abstract}
The video performance benefits of burst-by-burst adaptive modulation are studied, employing a higherorder modulation mode, when the channel is favourable, in order to increase the system's bits per symbol throughput and conversely, invoking a more robust, lower order modulation mode, when the channel exhibits inferior channel quality. It is shown that due to the proposed adaptive modem mode switching regime a seemless video-quality versus channel quality relationship can be established, resulting in error-free video quality right across the operating channel Signal-to-Noise Ratio (SNR) range.
\end{abstract}

\section{INTRODUCTION}

In burst-by-burst adaptive modulation a higher-order modulation scheme is invoked, when the channel is favourable, in order to increase the system's bits per symbol capacity and conversely, a more robust lower order modulation scheme is employed, when the channel exhibits inferior channel quality, in order to improve the mean Bit Error Ratio (BER) performance [1]-[4].

We found that the residual channel-induced inter-symbolinterference (ISI) at the output of the decision feedback equaliser (DFE) is near-Gaussian distributed and that if there are no decision feedback errors, the pseudo-SNR at the output of the DFE, $\gamma_{d f e}$ can be calculated as [5]:

$$
\begin{aligned}
\gamma_{d f e} & =\frac{\text { Wanted Signal Power }}{\text { Residual ISI Power }+ \text { Effective Noise Power }} \\
& =\frac{E\left[\left|S_{k} \sum_{m=0}^{N_{f}-1} C_{m} h_{m}\right|^{2}\right]}{\sum_{q=-\left(N_{f}-1\right)}^{-1} E\left[\left|f_{q} S_{k-q}\right|^{2}\right]+N_{o} \sum_{m=0}^{N_{f}-1}\left|C_{m}\right|^{2}},
\end{aligned}
$$

where $C_{m}$ and $h_{m}$ denote the DFE's feed-forward coefficients and the channel impulse response, respectively. The transmitted signal and the noise spectral density is represented by $S_{k}$ and $N_{o}$. Lastly, the number of DFE feedforward coefficients is denoted by $N_{f}$. By utilizing the

Key words: H.263, burst-by-burst adaptive modems, modulation, wireless video, video telephony

The financial support of the following organisations is gratefully acknowledged: Mobile Virtual Centre of Excellence, UK; Motorola ECID, Swindon, UK; European Commission, Bruxelles; EPSRC, Swindon, UK.
pseudo-SNR at the output of the equalizer, we are ensuring that the system performance is optimised by employing equalization and adaptive quadrature amplitude modulation [6] (AQAM)in a wideband environment according to the following switching regime:

$$
\text { Modulation Mode }= \begin{cases}B P S K & \text { if } \gamma_{D F E}<f_{1} \\ 4 Q A M & \text { if } f_{1}<\gamma_{D F E}<f_{2} \\ 16 Q A M & \text { if } f_{2}<\gamma_{D F E}<f_{3} \\ 64 Q A M & \text { if } \gamma_{D F E}>f_{3},\end{cases}
$$

where $f_{n}, n=1 \ldots 3$ are the pseudo-SNR thresholds levels, which are set according to the system's integrity requirements.

Having reviewed the background of burst-by-burst adaptive modems, we now focus our attention on video issues. Färber, Steinbach and Girod at Erlangen University contrived various error-resilient H.263-based schemes [8], while Sadka, Eryurtlu and Kondoz [9] from Surrey University proposed various improvements to the H.263 scheme. The philosophy of our proposed schemes follows that of the narrowband, statically configured multimode system introduced in [10], employing an adaptive rate control and packetisation algorithm, supporting constant Baud-rate operation. In this contribution we employed wideband burstby-burst adaptive modulation, in order to quantify the video performance benefits of such systems. It is an important element of the system that when the $\mathrm{BCH}$ codes protecting the video stream are overwhelmed by the plethora of transmission errors, we refrain from decoding the video packet in order to prevent error propagation through the reconstructed frame buffer [10]. Instead, these corrupted packets are dropped and the reconstructed frame buffer will not be updated, until the next packet replenishing the specific video frame area arrives. The associated video performance degradation is fairly minor for packet dropping or frame error rates (FER) below about $5 \%$. These packet dropping events are signalled to the remote decoder by superimposing a strongly protected onebit packet acknowledgement flag on the reverse-direction packet, as outlined in [10].

The paper is structured as follows. Section 2 introduces the video transceiver parameters, while the bulk of the paper is constituted by the video performance analysis of Section 3. Section 4 characterises the effects of adaptive modem mode switching thresholds on the system's video performance, before concluding in Section 5 . 


\begin{tabular}{|l|c|}
\hline Parameter & Value \\
\hline \hline Carrier Frequency & $1.9 \mathrm{GHz}$ \\
\hline Vehicular Speed & $30 \mathrm{mph}$ \\
\hline Doppler frequency & $85 \mathrm{~Hz}$ \\
\hline Normalised Doppler fr. & $3.27 \times 10^{-5}$ \\
\hline Channel type & COST 207 Typ. Urban (Figure 1) \\
\hline No. of paths in channel & Adaptive QAM \\
\hline Data modulation & (BPSK, 4-QAM, 16-QAM, 64-QAM) \\
\hline Receiver type & $\begin{array}{c}\text { Decision Feedback Equalizer } \\
\text { Number of Forward Filter Taps }=35 \\
\text { Number of Backward Filter Taps }=7\end{array}$ \\
\hline
\end{tabular}

\begin{tabular}{|l||c|}
\hline Features & Value \\
\hline \hline Multiple access & TDMA \\
\hline No. of Slots/Frame & 16 \\
\hline TDMA frame length & $4.615 \mathrm{~ms}$ \\
\hline TDMA slot length & $288 \mu \mathrm{s}$ \\
\hline Data Symbols/TDMA slot & 684 \\
\hline User Data Symbol Rate (KBd) & 148.2 \\
\hline System Data Symbol Rate (MBd) & 2.37 \\
\hline Symbols/TDMA slot & 750 \\
\hline User Symbol Rate (KBd) & 162.5 \\
\hline System Symbol Rate (MBd) & 2.6 \\
\hline System Bandwidth $(\mathrm{MHz})$ & 3.9 \\
\hline Eff. User Bandwidth $(\mathrm{kHz})$ & 244 \\
\hline
\end{tabular}

Table 1: Modulation and channel parameters

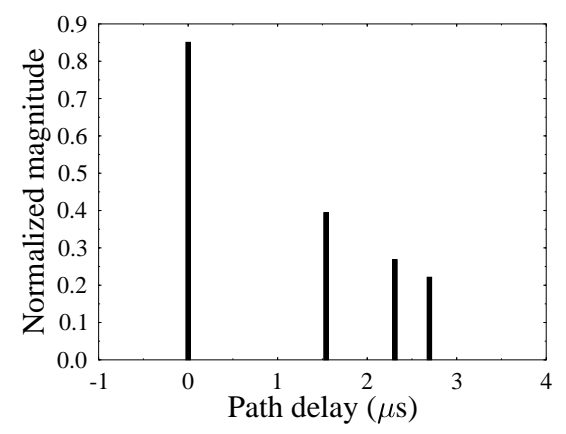

Figure 1: Normalized channel impulse response for the COST 207 [12] four-path Typical Urban channel.

\section{VIDEO TRANSCEIVER}

In this contribution we used $176 \times 144$ pixel QCIF-resolution, 30 frames/s video sequences encoded at bitrates resulting in high perceptual video quality. Table 1 shows the modulationand channel-parameters employed. The COST207 [12] fourpath typical urban (TU) channel model was used and its impulse response is portrayed in Figure 1. We used the Pan-European FRAMES proposal [11] as the basis for our wideband transmission system, the frame structure of which is shown in Figure 2. Employing the FRAMES Mode A1 (FMA1) so-called non-spread data burst mode required a system bandwidth of $3.9 \mathrm{MHz}$, when assuming a modulation excess bandwidth of $50 \%$. A range of other system parameters are shown in Table 2.

The proposed video transceiver is based on the H.263

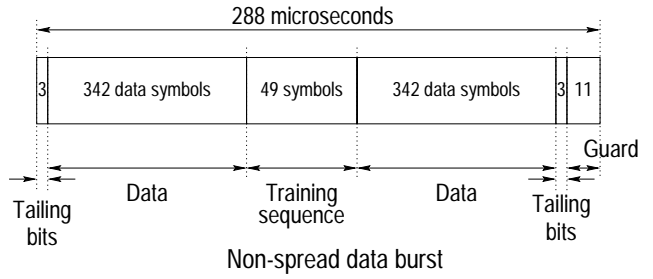

Figure 2: Transmission burst structure of the FMA1 nonspread data burst mode of the FRAMES proposal [11]
Table 2: Generic system features of the reconfigurable multi-mode video transceiver, using the non-spread data burst mode of the FRAMES proposal [11] shown in Figure 2 .

\begin{tabular}{|l||c|c|c|c|}
\hline \multicolumn{1}{|l||}{ Features } & \multicolumn{4}{c|}{ Multi-rate System } \\
\hline \hline Mode & BPSK & 4QAM & 16QAM & 64QAM \\
\hline Bits/Symbol & 1 & 2 & 4 & 6 \\
\hline FEC & \multicolumn{4}{|c|}{ Near Half-rate BCH } \\
\hline $\begin{array}{l}\text { Transmission } \\
\text { bitrate (kbit/s) }\end{array}$ & 148.2 & 296.4 & 592.8 & 889.3 \\
\hline $\begin{array}{l}\text { Unprotected } \\
\text { bitrate (kbit/s) }\end{array}$ & 75.8 & 151.7 & 303.4 & 456.1 \\
\hline $\begin{array}{l}\text { Effective } \\
\text { Video-rate (kbit/s) }\end{array}$ & 67.0 & 141.7 & 292.1 & 446.4 \\
\hline Video fr. rate (Hz) & & \multicolumn{4}{|c|}{30} \\
\hline
\end{tabular}

Table 3: Operational-mode specific transceiver parameters

video codec [13]. The video coded bitstream was protected by binary Bose-Chaudhuri-Hocquenghem $(\mathrm{BCH})$ coding [14] combined with an intelligent burst-by-burst adaptive wideband multi-mode Quadrature Amplitude Modulation (QAM) modem [6], which can be configured either under network control or under transceiver control to operate as a 1, 2, 4 and 6 bits/symbol scheme, while maintaining a constant signalling rate. This allowed us to support an increased throughput expressed in terms of the average number of bits per symbol, when the instantaneous channel quality was high, leading ultimately to an increased video quality in a constant bandwidth.

The transmitted bitrate for all four modes of operation is shown in Table 3. The unprotected bitrate before approximately half-rate $\mathrm{BCH}$ coding is also shown in the table. The actual useful bitrate available for video is slightly less, than the unprotected bitrate due to the required strongly protected packet acknowledgement information and packetisation information. The effective video bitrate is also shown in the table.

\section{BURST-BY-BURST ADAPTIVE VIDEOPHONE PERFORMANCE}

Figure 3 demonstrates how the burst-by-burst adaptive modem changes its modulation modes every transmission burst, ie every $4.615 \mathrm{~ms}$, based on the fluctuating pseudo-SNR. The right-hand-side vertical axis indicates the associated 


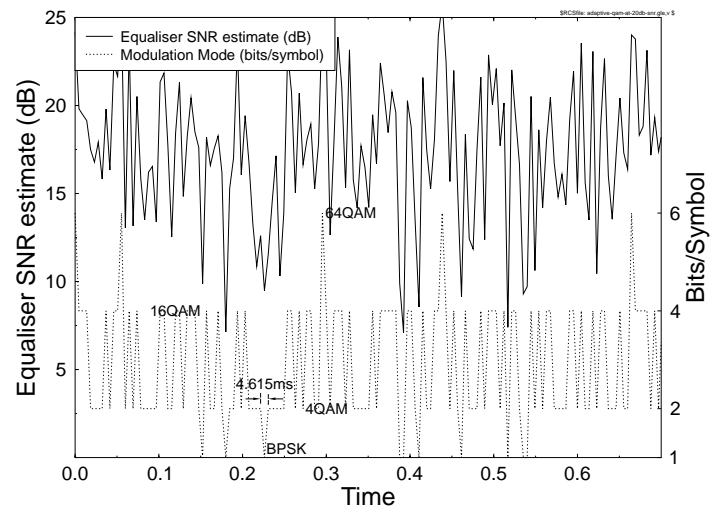

Figure 3: Adaptive burst-by-burst modem in operation for an average channel SNR of $20 \mathrm{~dB}$, where the modulation mode switching is based upon the SNR estimate at the output of the equaliser, using the channel parameters defined in Table 1.

number of bits per symbol.

In order to judge the benefits of burst-by-burst adaptive modulation we considered two scenarios. In the first scheme the adaptive modem always chose the perfectly estimated AQAM modulation mode, in order to provide a maximum upper bound performance. In the second scenario the modulation mode was based upon the perfectly estimated AQAM modulation mode for the previous burst, which corresponded to a delay of one Time Division Multiple Access (TDMA) frame duration of $4.615 \mathrm{~ms}$. This second scenario represents a practical burst-by-burst adaptive modem, where the one-frame channel quality estimation latency is due to superimposing the receiver's perceived channel quality on a reverse-direction packet, for informing the transmitter concerning the best modem mode to be used.

Figure 4 shows the transmission FER (or packet loss ratio) versus channel SNR for the 1, 2, 4 and $6 \mathrm{bit} / \mathrm{symbol}$ fixed modulation schemes, as well as for the ideal and for the one-frame delayed realistic scenarios using the burstby-burst adaptive QAM (AQAM) modem. A somewhat surprising fact is [7] - which is not explicitly shown here due to lack of space - that at low SNRs AQAM can maintain a lower BER than BPSK, since under inferior instantaneous channel conditions it exhibits the corresponding BPSK BER, but the average number of transmitted AQAM bits is higher than that of BPSK, resulting in a reduced average AQAM BER. At high SNRs the associated AQAM BER curve - which is not shown here due to lack of space asymptotically joins the 64QAM BER curve, since 64QAM is the predominant mode used. The corresponding FER curve of Figure 4 obeys similar tendencies in terms of having the BPSK and 64QAM FER curves as asymptotes at low and high SNRs, respectively. However - in contrast to the BER - the AQAM FER cannot be lower than that of BPSK, since the same number of frames is transmitted in both cases. The substantial advantage of AQAM is that due to its higher number of bits/symbol - the number bits transmitted per frame is higher, resulting in an increased video quality. ${ }^{1}$. The same FER curves are also portrayed

\footnotetext{
${ }^{1}$ We note here that the associated performance results typi-
}

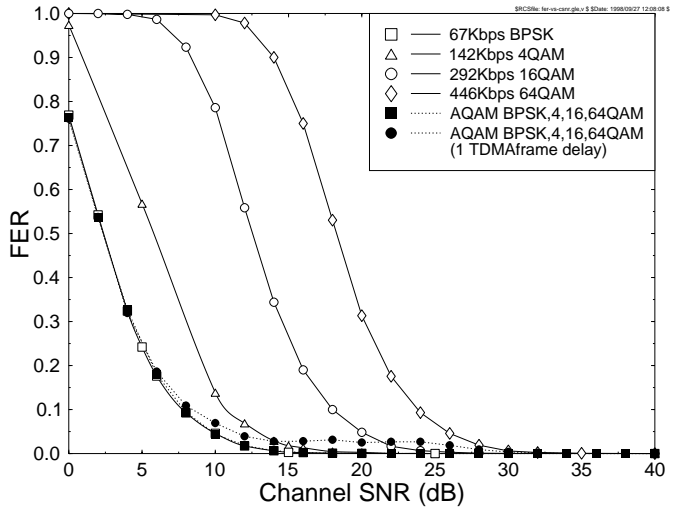

Figure 4: Transmission FER (or packet loss ratio) versus Channel SNR comparison of the four fixed modulation modes (BPSK, 4QAM, 16QAM, 64QAM) and that of the adaptive burst-by-burst modem (AQAM). AQAM is shown with a realistic one TDMA frame delay between channel estimation and mode switching, and also with a zero delay version for indicating the upper bound performance. The channel parameters were defined in Table 1.

on a logarithmic scale in Figure 5, where - for the sake of comparison - we showed the associated FER curve for statically reconfigured modem modes switching at $5 \%$ transmission FER, as it will be detailed below.

Explicitly, the statically reconfigured modem was invoked in Figure 5 as a benchmarker, in order to indicate, how a system would perform, which cannot act on the basis of the near-instantaneously varying channel quality. As it can be inferred from Figure 5, such a statically reconfigured transceiver switches its mode of operation from a lower-order modem mode, such as for example BPSK to a higher-order mode, such as 4QAM, when the channel quality has improved sufficiently for the 4QAM mode's FER to become lower than $5 \%$ upon reconfiguring the transceiver in this 4QAM mode. Again - as seen also in Figure 4 earlier on a non-logarithmic scale - Figure 5 clearly shows on a logarithmic scale that the 'one-frame channel estimation delay' AQAM modem manages to maintain a similar FER performance to the fixed rate BPSK modem at low SNRs, despite the fact that AQAM provides increasingly higher bitrates, reaching four times higher values than BPSK for high channel SNRs, where the employment of 64QAM is predominant. In this high SNR region the FER curve asymptotically approaches the 64QAM FER curve for both the realistic and the ideal AQAM scheme, although this is not visible in the figure for the ideal scheme, since this occures at SNRs outside the range of Figure 5. Again, the reason for this performance discrepancy is the occasionally misjudged channel quality estimates of the realistic AQAM scheme. Additionally, Figure 5 indicates that the realistic AQAM modem exhibits a near-constant $3 \% \mathrm{FER}$ at medium SNRs. The issue of adjusting the switching thresholds in oder to achieve the target FER will be addressed in detail in Section 4 and the thresholds invoked will be detailed with reference to

cally degrade upon increasing the Doppler frequency and improve upon reducing it, since the effects of channel estimation latency become less significant. This phenomenon was quantified in [7] 


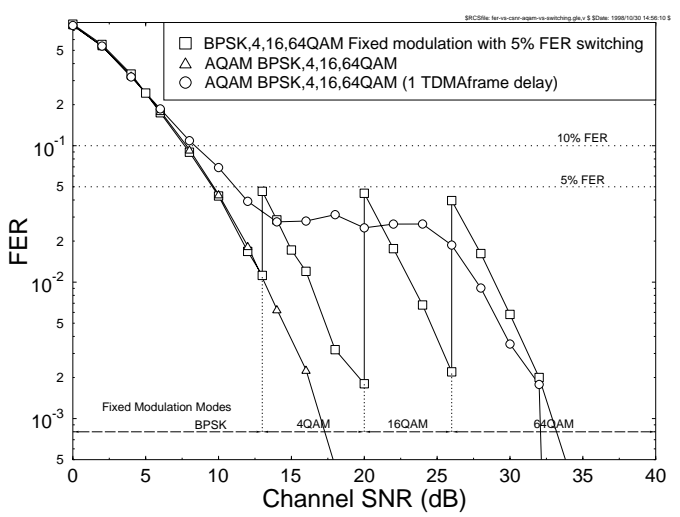

Figure 5: Transmission FER (or packet loss ratio) versus Channel SNR comparison of the four fixed modulation modes (BPSK, 4QAM, 16QAM, 64QAM) with 5\% FER switching and adaptive burst-by-burst modem (AQAM). AQAM is shown with a realistic one TDMA frame delay between channel estimation and mode switching, and a zero delay version is included as an upper bound. The channel parameters were defined in Table 1.

Table 4. Suffice to say at this stage that the average number of bits per symbol - and potentially also the associated video quality - can be increased upon using more aggressive switching thresholds. However, this results in an increased FER, which tends to decrease the video quality, as it will be discussed in Section 4.

Let us now demonstrate these effects on the decoded video quality, measured in terms of the Peak Signal-to-Noise Ratio (PSNR). Figure 6 shows the decoded video quality in terms of PSNR versus channel SNR for both the ideal and realistic adaptive modem, and for the four modes of the statically configured multi-mode modem. It can be seen that the ideal adaptive modem, which always selects the perfect modulation modes, has a better or similar video quality for the whole range of channel SNRs. For the statically configured multi-mode scheme the video quality degrades, when the system switches from a higher-order to a lower-order modulation mode. The ideal adaptive modem however smoothes out the sudden loss of video quality, as the channel degrades. The non-ideal adaptive modem has a slightly lower video quality performance, than the ideal adaptive modem, especially at medium SNRs, since it sometimes selects the incorrect modulation mode due to the estimation delay. This can inflict video packet loss and/or a reduction of the effective video bitrate, which in turn reduces the video quality.

\section{SWITCHING THRESHOLDS}

The burst-by-burst adaptive modem changes its modulation modes based on the fluctuating channel conditions expressed in terms of the SNR at the equaliser's output. The set of switching thresholds used in all the previous graphs is the "Standard" set shown in Table 4, which was determined on the basis of the required channel SINR for maintaining the specific target video FER.

In order to investigate the effect of different sets of

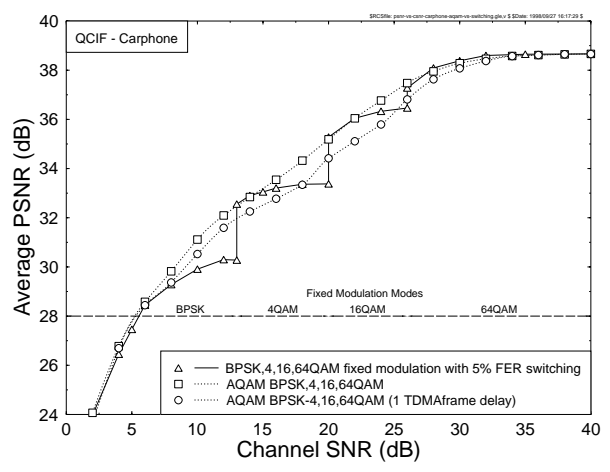

Figure 6: Decoded video quality (PSNR) versus channel SNR comparison of the four fixed modulation modes (BPSK, 4QAM, 16QAM, 64QAM) with 5\% transmission FER switching and that of the adaptive burst-by-burst modem (AQAM). AQAM is shown with a realistic one TDMA frame delay between channel estimation and mode switching, and a zero delay version for indicating the upper bound. The channel parameters were defined in Table 1 .

\begin{tabular}{|l|c|c|c|c|}
\hline & BPSK & $4 \mathrm{QAM}$ & $16 \mathrm{QAM}$ & $64 \mathrm{QAM}$ \\
\hline \hline Standard & $<10 \mathrm{~dB}$ & $\geq 10 \mathrm{~dB}$ & $\geq 18 \mathrm{~dB}$ & $\geq 24 \mathrm{~dB}$ \\
\hline Conservative & $<13 \mathrm{~dB}$ & $\geq 13 \mathrm{~dB}$ & $\geq 20 \mathrm{~dB}$ & $\geq 26 \mathrm{~dB}$ \\
\hline Aggressive & $<9 \mathrm{~dB}$ & $\geq 9 \mathrm{~dB}$ & $\geq 17 \mathrm{~dB}$ & $\geq 23 \mathrm{~dB}$ \\
\hline
\end{tabular}

Table 4: SINR estimate at output of the equaliser required for each modulation mode in Burst-by-Burst Adaptive modem, ie. switching thresholds

switching thresholds, we defined two new sets of thresholds, a more conservative set, and a more aggressive set, employing less robust, but more bandwidth-efficient modem modes at lower SNRs. The more conservative switching thresholds reduced the transmission FER at the expense of a lower effective video bitrate. The more aggressive set of thresholds increased the effective video bitrate at the expense of a higher transmission FER. shown in Figure 7. The more conservative set of switching thresholds reduces the effective video bitrate but also reduces the transmission FER. The aggressive switching thresholds, increase the effective video bitrate, but also increase the transmission FER. Therefore the optimal switching thresholds should be set such that the transmission FER is deemed acceptable is the range of channel SNRs considered.

\section{CONCLUSIONS}

In this contribution we have proposed a wideband burts-byburst adaptive modem, which employs the pseudo-SNR perceived by the channel equaliser as the quality measure for controlling the modem modes. Furthermore, we have quantified the achievable video performance gains due to employing the proposed wideband burts-by-burst adaptive modem. When our adaptive packetiser is used in conjunction with the adaptive modem, it continually adjusts the video codec's target bitrate to match the instantaneous bitrate capacity provided by the adaptive modem. The proposed burst-by-burst adaptive video transceiver exhibits a better 


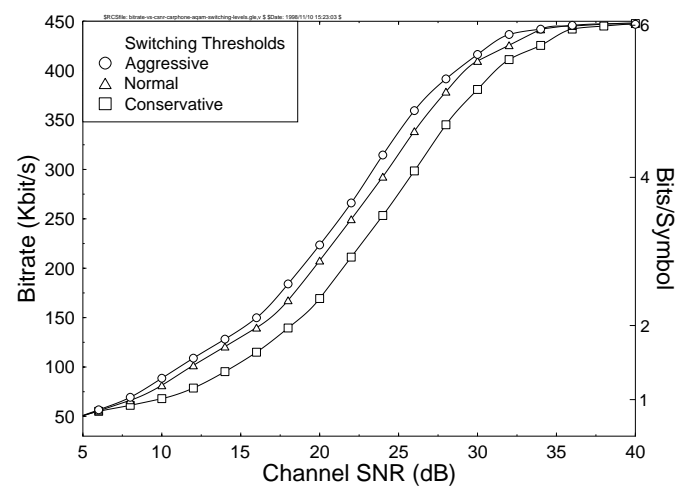

Figure 7: Video bitrate versus channel SNR comparison for the adaptive burst-by-burst modem (AQAM) with a realistic one TDMA frame delay between channel estimation and mode switching for the three sets of switching thresholds as described in Table 4. The channel parameters were defined in Table 1.

video performance, than a statically configured multi-mode scheme. Our future work will be concentrated on improving the system performance by invoking more complex turbo coding schemes.

\section{REFERENCES}

[1] W.T. Webb and R. Steele, "Variable Rate QAM for mobile radio," IEEE Transactions on Communications, vol. 43, pp. 2223 - 2230, July 1995.

[2] J.M. Torrance and L. Hanzo, "On the Upper bound performance of adaptive QAM in a slow Rayleigh fading," IEE Electronics Letters, pp. 169 - 171, April 1996.

[3] H. Matsuako, S. Sampei, N. Morinaga and Y. Kamio, "Adaptive Modulation systems with variable coding rate concatenated code for high quality multi-media communication systems," in Proceedings of IEEE Vehicular Technology Conference, pp. 487 - 491, 1996.

[4] A. J. Goldsmith and S. G. Chua, "Variable Rate Variable Power MQAM for Fading Channels," IEEE Transactions on Communications, vol. 45, pp. 1218 1230, October 1997.

[5] J. C. Cheung, Adaptive Equalisers for Wideband TDMA Mobile Radio. PhD thesis, University of Southampton, 1991.

[6] W. T. Webb and L. Hanzo, Modern Quadrature Amplitude Modulation: Principles and Applications for Wireless Communications. IEEE Press-Pentech Press, 1994. ISBN 0-7273-1701-6.

[7] J. M. Torrance, L. Hanzo: Latency and Networking Aspects of Adaptive Modems over Slow Indoors Rayleigh Fading Channels, IEEE Tr. on Veh. Techn., July 1999, Vol. 48, No. 4, pp 1237-1251

[8] N. Faerber, E. Steinbach, B. Girod: "Robust H.263 Compatible Transmission for Mobile Video Server Access", Proc. First International Workshop on Wireless
Image/Video Communications, Loughborough University, UK, 4-5 September 1996, pp 8-13

[9] A.H. Sadka, F. Eryurtlu, A.M. Kondoz: Improved performance H.263 under erroneous transmission conditions, Electr. Letters, 16th of Jan., 1997, Vol. 33, No. 2, pp 122-124

[10] P. Cherriman, L. Hanzo: Programable H.263-based wireless video transceivers for interference-limited environments, IEEE Tr. on CSVT, June 1997, Vol. 8, No.3, pp 275-286

[11] A. Klein, R. Pirhonen, J. Skoeld, and R. Suoranta, "FRAMES multiple access mode 1 - wideband TDMA with and without spreading," in Proceedings of IEEE International Symposium on Personal, Indoor and Mobile Radio Communications, PIMRC'97, vol. 1, (Marina Congress Centre, Helsinki, Finland), pp. 37-41, IEEE, 1-4 Sept 1997.

[12] "COST 207: Digital land mobile radio communications, final report." Office for Official Publications of the European Communities, 1989. Luxembourg.

[13] ITU-T, Recommendation H.263: Video coding for low bitrate communication, March 1996.

[14] R. Steele, L. Hanzo (Ed.), Mobile Radio Communications, 2nd edition, IEEE Press-John Wiley and Sons Ltd., 1999 